\title{
Resistance and susceptibility to weight gain: Individual variability in response to a high-fat diet
}

\author{
J.E. Blundell ${ }^{\text {a,* }}$, R.J. Stubbs ${ }^{\text {b }}$, C. Golding ${ }^{\text {a }}$, F. Croden ${ }^{\text {a }}$, R. Alam ${ }^{\text {a }}$, S. Whybrow ${ }^{\mathrm{b}}$, \\ J. Le Noury ${ }^{\mathrm{a}}$, C.L. Lawton ${ }^{\mathrm{a}}$ \\ a Institute of Psychological Sciences, University of Leeds, Leeds, LS2 9JT, UK \\ ${ }^{\mathrm{b}}$ Human Nutrition Laboratory, Rowett Research Institute, Aberdeen, UK
}

Received 15 August 2005; accepted 25 August 2005

\begin{abstract}
An obesigenic environment is a potent force for promoting weight gain. However, not all people exposed to such an environment become obese; some remain lean. This means that some people are susceptible to weight gain (in a weight-promoting environment) and others are resistant. Identifying the characteristics of appetite control and food motivation in these two groups could throw light on the causes of weight gain and how this can be either treated or prevented. We have investigated the issue experimentally by identifying people who habitually consume a high-fat diet (greater than $43 \%$ fat energy). These individuals have been termed high-fat phenotypes. We have compared individuals, of the same age $($ mean $=37$ years old) and gender (male), who have gained weight $(B M I=34)$ or who have remained lean $(B M I=22)$. The susceptible individuals are characterised by a cluster of characteristics including a weak satiety response to fatty meals, a maintained preference for high-fat over lowenergy foods in the post-ingestive satiety period, a strong hedonic attraction to palatable foods and to eating, and high scores on the TFEQ factors of Disinhibition and Hunger. The analysis of large databases suggests that this profile of factors contributes to an average daily positive energy balance from food of approximately $0.5 \mathrm{MJ}$. This profile of characteristics helps to define the symptomatology of a thrifty phenotype.
\end{abstract}

(C) 2005 Elsevier Inc. All rights reserved.

Keywords: Weight gain; Obesity; Satiety; TFEQ disinhibition; Dietary fat; Hunger; Behavioral phenotypes

\section{Background}

A large number of factors have been shown to be associated with high BMI or an increase in body weight. These include factors associated with food itself, for example, high-energy density, high-fat, portion size, sweet-fat combination, sugary drinks, etc., and features of the food environment, for example, eating in fast-food restaurants, food eaten outside the home, snacking and TV viewing, and others. The obesigenic environment is also characterised by abundant availability, easy accessibility, and aggressive marketing of foods. However, despite the potency of the obesigenic environment not all people become obese; some remain lean. This means that some people are susceptible to weight gain (in a weight-promoting environment) and others are resistant. The premise underlying this approach is that it is possible to characterise the psycho-

\footnotetext{
* Corresponding author. Tel.: +44 1133435742.

E-mail address: J.E.Blundell@Leeds.ac.uk (J.E. Blundell).
}

biological variability (physiological signalling, metabolic processes, psychological responsiveness, traits and states) to identify factors which make some people susceptible to weight gain (via appetite-regulating processes) and others resistant. It follows from this that, in response to some environmental trigger, there will not be a uniform response from all individuals. Instead, the response will be variable and will reflect biological variability. Studying this variability can reveal processes of individual weight regulation.

This argument is not weakened by the demonstrations that a number of food-related manipulations concerning energy density or portion size cause subjects to over-consume in experimental settings. Despite the potency of the food environment manipulation, intrinsic signals do come into play so that people do stop eating at a certain point. In addition, the strength of these signals (and their operation upon eating behaviour) varies from person to person. Therefore, individual variability is still apparent in the amount consumed even during certain carefully contrived experimental manipulations. 
Fig. 1 theoretically illustrates how the potential for weight gain is expressed in an obesigenic environment, but obscured in a restrictive environment [1]. The plethora of opportunities for consumption (and over-consumption) in an obesigenic environment allow the variable strengths of genetic (biological) dispositions to be readily disclosed.

\section{Risk factors for weight gain-via appetite}

Most researchers do not have any trouble accepting the idea that the state of a person's metabolism constitutes a major risk for developing weight gain and becoming obese. However, as obesity develops, metabolic characteristics change so that the state of obesity itself is associated with a different metabolic profile to that accompanying the process of weight gain. This makes it important to do longitudinal studies (whilst weight is increasing) as well as cross-sectional studies (comparing lean and obese subjects). Recently, Ravussin and Kozak [2] have drawn attention to this issue and have outlined those metabolic and physiological factors associated with weight gain and following the attainment of the obese state.

The tendency to gain weight is associated with a low basal metabolic rate, low energy cost of physical activity, a low capacity for fat oxidation (relatively high respiratory quotientRQ), high insulin sensitivity, low sympathetic nervous system activity and a low plasma leptin concentration. In the state of obesity itself, many of these risk factors (or predictors of weight gain) are reversed.

Just as certain metabolic variables (risk factors) can lead to a positive energy balance, we can envisage certain behaviourally mediated processes which themselves constitute the risk factors leading to hyperphagia or 'over-consumption' (high-energy intake leading to a positive energy balance). These processes may be patterns of eating behaviour, the sensory or hedonic events which guide behaviour, or sensations which accompany or follow eating. For convenience, this cluster of events can be referred to as behavioural risk factors. These events may include a preference for fatty foods, weakened satiation (end of meal signals), relatively weak satiety (post-ingestive inhibition over further eating), strong oro-sensory preferences (e.g., for sweetness combined with fattiness in foods), a binge potential, and a

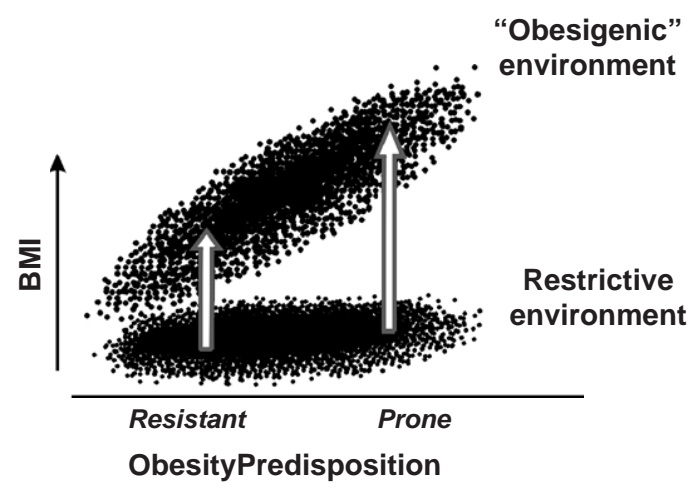

Fig. 1. Potential effects of genetic background (obesity disposition) in individuals defined as resistant or prone, in a restrictive or 'obesigenic' environment. After Ravussin and Kozak [2]. high food-induced pleasure response. In turn, these events may be sub-divided to describe more specific components leading to a risk of over-consumption.

These behavioural risk factors can be regarded as biological dispositions which create vulnerability for weight gain and which manifest themselves through behavioural acts themselves, or through physiological processes which promote or permit changes in behaviour. This conceptualisation is set out in Table 1.

However, such risk factors alone would be unlikely to lead to a positive energy balance in a benign environment; that is, one in which the food supply and the cultural habits worked against excessive consumption. In most of today's societies, however, the food environment exploits biologically based dispositions and this promotes the attainment of a high-energy intake.

\section{Components of susceptibility}

The notion that some people are more susceptible to weight gain than others is a truism. The issue is how to characterise the features of susceptibility and then, to decide how these features can be used to deal with the epidemic of obesity. Susceptibility can be identified at various levels - genetic, physiologic and metabolic, behavioural and psychological. Identifying specific genes or allelic variations that are associated with obesity or body weight gain is already well advanced $[3,4]$. A small number of single gene mutations have been identified that can lead to obesity. The most common is the mutation of the MC4-R which may account for about $4 \%$ of adult obesity [5]. However, it is widely agreed that obesity in most people is under polygenic influence. Both linkage studies and candidate gene approaches have identified a large number of genes whose variants are more strongly associated with obese individuals than with lean people. However, it is clearly likely that the spectrum of 'obesityrelated' genes will vary among individuals so that not all people have the some profile of genetic susceptibility. Equally, the same degree of susceptibility to obesity may be conferred by different profiles of allelic variation in a variable number of genes. However, under these circumstances, the characteristics of susceptibility should be qualitatively different. This issue draws attention to the obvious fact that there are many routes to obesity, and that people gaining weight carry with them a variable array of susceptibility factors.

A second level of susceptibility-which has implications for the control of eating in response to diet-is the central neurochemical and the peripheral physiological mechanisms that are involved in homeostatic and hedonic processes. Differences in the release or sustained levels of biologically important molecules such as insulin, cholecystokinin (CCK), glucagon-like-peptide (GLP-1), peptide YY (P-YY), leptin, adiponectin and ghrelin have all been proposed to have a potential role in the control of physiological processes of satiation or satiety, or in the behavioural end points of meal size or meal frequency $[6,7]$. Consequently, susceptibility to overeating (poor control of satiation or satiety) is widely believed to be conferred by variable levels in plasma or at target sites of a number of peripheral signalling molecules. Biological variability among individuals and different profiles of signalling molecules will automatically 
Table 1

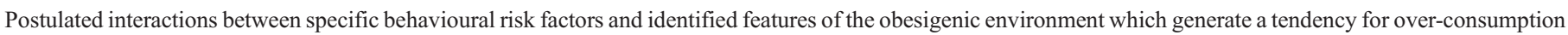

\begin{tabular}{|c|c|c|}
\hline Biological vulnerability & Environmental influence & Potential for over-consumption \\
\hline Preference for fatty foods & Abundance of high-fat food (high-energy dense) & Fat intake \\
\hline Weak satiation (end of meal signals) & Large portion sizes & Meal size \\
\hline Oro-sensory responsiveness & Availability of highly palatable foods with specific sensory-nutrient combinations & $\begin{array}{ll}\uparrow & \text { Amount eaten } \\
\uparrow & \text { Frequency of eating }\end{array}$ \\
\hline Weak post-ingestive satiety & Easy accessibility to foods and presence of potent priming stimuli & $\begin{array}{ll}\uparrow & \text { Frequency of eating } \\
\uparrow & \text { Tendency to re-initiate eating }\end{array}$ \\
\hline
\end{tabular}

Modified from Blundell and Cooling [23].

confer the capacity for strong or weak control of satiety in response to specific nutritional inputs. These peptides are often referred to as biomarkers of satiation and satiety [8].

Currently, most information concerning the physiology of satiety in humans relates to the peripheral system - for obvious reasons. However, extrapolation from animal studies, together with some evidence from genetic mutations, strongly suggests a matrix of signals in the human brain that organize the homeostatic processes controlling the excitation or inhibition of eating [7,9].

Although homeostasis is the predominant conceptual position for studying the control of food intake, in recent years there has been considerable interest in hedonic processes. This has given rise to comparisons between homeostatic and hedonic systems [10] and to a questioning of the relative potency of homeostatic and hedonic risk factors for over-consumption $[11,12]$. Indeed it has been argued that the ubiquitous presence of highly palatable food in the environment could chronically activate the hedonic system which, in the absence of any distinct homeostatic need, would promote consumption and weight gain [13]. In the homeostatic approach to appetite control, the emphasis is on processes subserving hunger and fullness, in which drives, arising from biological needs, are balanced by physiological satiety signalling systems. The hedonic component of eating is commonly regarded as the feeling of pleasure that is associated with eating. However, it is more complicated than this and involves the concepts of incentive value and reward value of foods. The dual system of 'liking' and 'wanting' [14] which has an identity in separate neural systems-has been referred to as the major driving force for ingestion [15], and the reward can override the metabolic regulatory system. A biological substrate mediating hedonic processes has also been investigated and includes a largely separate set of brain neurotransmitters including glutamate, opioids, endocannabinoid and dopamine pathways and associated receptor systems. Together, these circuits are probably responsible for the biological basis of processes such as wanting and liking that are associated with the selection and consumption of foods [14].

One immediate question concerns the extent to which the homeostatic and hedonic components are responsible for the development of weight gain and obesity. Are individuals who are susceptible to weight gain characterised by defects in satiety signalling and chronic high levels of hunger? Or are they characterised by an overwhelming hyper-responsivity to the perceived pleasantness of foods? Pharmacological evidence suggests that the substrates are rather separate. For example, in obese subjects, administration of the serotonin drug D-fenfluramine [16] suppressed the sensation of hunger and had no effect on the appreciation of the pleasantness of food. Conversely, nalmafene, an opioid agonist, reduced the rated pleasantness of palatable foods but had no effect on hunger [17].

A third level of susceptibility relates to behavioural and psychological characteristics. The model depicted in Table 1 related these characteristics to underlying biological vulnerability, but the behavioural and psychological features can be defined separately. One fundamental behavioural feature is the pattern of eating defined by the distribution of eating episodes across the day, and the types of foods selected for consumption. It can probably be agreed that individuals susceptible to weight gain could be characterised by large eating episodes (big meal sizes), by more frequent eating episodes (meals plus snacks) or by preferential selection of high-fat or high-energy density foods (or combinations of all of these). Together, these features would inevitably lead to a high daily energy intake, which, if not compensated for by an adjustment in some other component of energy balance system, would lead to a positive energy balance and weight gain. In principle, it should be possible to describe the behavioural patterns and food choice profiles of susceptible or resistant individuals to identify differences. However, given the difficulty of obtaining valid data under free-living conditions (e.g., [18]), the expression of behaviour or food choice is more frequently sampled under laboratory conditions. The data captured under these circumstances is normally accurate and precise, but it is clearly not natural. This means that the measurements of behavioural susceptibility will normally reflect a compromise between naturalness and precision. Of course, under laboratory conditions, it is usually possible to experimentally interrogate the system to provide instances of the strength of compensation to over- or under-feeding, the potency of carefully monitored post-ingestive processes, or the interaction between homeostatic ad hedonic influences.

\subsection{States and traits}

It is well recognized that the behavioural acts of eating and food selection are usually accompanied by subjective states which, some people argue, actually cause the behaviour. For example, a strong perceived hunger may lead to rapid onset of eating and a large meal size. However, in considering the psychological aspects of eating motivation, it is theoretically appropriate to distinguish between states and traits. In this context, states can be identified as those sensations such as 
hunger, fullness and wanting that oscillate episodically and which are primarily entrained with the pattern of eating. The fluctuation of these states can be described by a certain frequency, amplitude and rhythmicity as exemplified by the diurnal profile of hunger. In contrast, traits represent more enduring and resilient influences on behaviour and do not fluctuate within a day or on a day-to-day basis. Such traits can be identified, for example, by the factors revealed by the ThreeFactor Eating Questionnaire (TFEQ) [19]. These traits have a tonic, rather than episodic, form of expression and they influence the tendency to eat or to select foods on a habitual long-term basis. States and traits, therefore, influence consumption in different ways and through different processes. When investigating the characteristics of susceptibility and resistance, it is therefore necessary for the methodology to incorporate measurements of both states and traits.

\subsection{Appetite and energy balance}

It is clear that factors associated with susceptibility and resistance do not reside solely in the domain of energy intake. A consideration of the energy balance concept readily illustrates how a tendency to gain weight could arise from a relatively low metabolic rate, weak thermogenic response to food, or a considerably reduced level of volitional physical activity arising from a low frequency of active behaviours or a preponderance of time spent in sedentary activities. Indeed, the concept of a generally conservative physiology-arising from a specific genetic profile-would imply that susceptibility to weight gain would entail both a tendency to conserve energy (through metabolic or behavioural processes), and a tendency to enhance energy intake. This argument follows the lines of the thrifty genotype concept [20]. It is worth keeping in mind that, whereas energy expenditure involves a variable proportion of behaviour (say $10-60 \%$ depending on the amount of physical activity undertaken), energy intake is $100 \%$ behaviour. Given these figures, it is clear that the expression of behaviour constitutes a major vehicle for susceptibility or resistance to weight gain.

\section{Functional phenotype approach}

There exist many mechanisms through which an individual could gain weight and become obese. As noted above, this diversity is reflected at the level of analysis of genetics, central and peripheral physiology, and the behavioural and psychological profile. It is also clear that a great number of environmental features can exploit intrinsic risk factors (see Table 1) to induce susceptibility. In recent years, dietary features, such as highenergy dense foods, high-fat, high-carbohydrate, high-GI, high intake of sweet beverages, high-sucrose, high-fructose corn syrup and highly palatable foods, plus various combinations of these, have been proposed as causal agents provoking susceptibility to weight gain. Consequently, in studying susceptibility, the unfettered operation of a plethora of dietary variables could easily occlude the disclosure of key factors. Therefore, in order to reduce the complexity of this nutritional environment, and to allow non-nutrient related features of susceptibility to be more easily identified, we have worked with individuals defined according to their habitual consumption of a particular type of diet. Such individuals have been called phenotypes.

In principle, a number of behavioural phenotypes could be defined based on taste preferences, patterns of eating, or motivational responses [21]. Because of the importance of dietary fat to energy balance and body weight regulation [22], researchers have paid attention to the habitual consumption of high-fat or low-fat foods. These have now been well defined and can be termed phenotypes [23]. The phenotype is defined by a particular pattern of behaviour characterised by the selection of high-fat or low-fat food items.

\subsection{The use of behavioural (dietary) phenotypes}

The approach described here is based on the identification of behavioural phenotypes. A phenotype is normally defined as a stable cluster of measurable characteristics that separate one 'type' from another. This 'type' is classically regarded as the consequences of a particular genotype. The approach therefore lends itself to the development of taxonomy of unambiguously defined 'types'; it is therefore a typology. In principle, a number of phenotypes could be defined based on objective or subjective aspects of appetite control. The approach favoured here emphasises behaviour since, in principle, this can be defined more rigorously and unambiguously than attitudes or subjective perceptions.

The power of this approach resides in the capacity to make clear distinctions among different phenotypes - that is, among groups of individuals with contrasting and habitual patterns of food selection. In turn the term selection is defined rigorously by reference to foods which are actually consumed. These phenotypes can be distinguished according to habitual dietary intakes (of foods which have been 'selected').

\subsection{High- and low-fat phenotypes}

High- (HF) and low-fat (LF) phenotypes are classified according to the type of diet habitually consumed; measured by a Food Frequency Questionnaire (FFQ) and diary record, and with under-reporters excluded. The records indicate that these groups habitually consume different types of foods and display different patterns of eating [24]. The research strategy is designed to allow individuals characterised in a population by their macronutrient intakes to be tracked so that their particular food choices and consumption of specific foods can be identified.

In principle, phenotypes can be identified independently of age or sex. However, in a first series of studies, the characteristics of young adult males have been examined. When subjected to energy and macronutrient challenges in order to evaluate the responses of the appetite control system, clear differences between the groups were demonstrated. Initially, HF displayed higher initial hunger levels, with a much sharper decline in hunger in response to meals or nutrient loads [25]. After eating, hunger recovered more rapidly in HF compared with LF. In addition, the size of a test meal consumed was closely related to 
the suppression of hunger in HF; in contrast, the appetite response system in LF appeared to be somewhat insensitive and damped. This relationship between habitual fat intake and hunger is reminiscent of a previous finding. French et al. [26] found that during 2 weeks of high-fat overfeeding to normalweight subjects, which caused a significant gain in weight, subjects displayed a progressive increase in hunger and a decrease in fullness before a test meal. Taken together these findings may indicate that eating a high-fat diet may facilitate feelings of hunger.

A further feature of these behaviour studies was that HF and LF differed in the control over meal size when offered an unlimited range of either high-fat or high-carbohydrate foods. HF consumed a similar weight of food on both diets, and therefore took in a much higher amount of energy with the high-fat (high-energy dense) foods. In contrast, LF consumed a much smaller amount of the high-fat foods, and consequently took in a similar amount of energy on both diets. These findings suggest that signalling systems for meal termination (satiation) and post-meal inhibition of appetite (satiety) operate with differing strengths in HF and LF. This finding may not be surprising in view of the fact that the gastrointestinal tract has become adapted to dealing with quite different dietary components, and this factor will have exerted a priming effect on specific satiety signals.

The existence of distinctive profiles of appetite control in HF and LF indicates different patterns of physiological responses to food ingestion. The possibility of other physiological differences was investigated using indirect calorimetry to measure BMR, $\mathrm{RQ}$ and dietary-induced thermogenic responses to specific fat and carbohydrate loads [27].

The results indicated that HF have a lower RQ than LF; this finding confirmed that fat oxidation was higher in HF, as would be expected due to the habitual high intake of fat-containing foods. However, an unexpected finding was the significantly higher BMR in HF than LF, together with different profiles of 'thermogenic' responses to the high-fat and high-carbohydrate loads. A further important finding was that HF had higher plasma leptin levels than LF [28] despite having similar levels of body fat.

However, the notion of individuals with different BMRs is consistent with the concept of 'energy-sparing' and 'energyprofligate' individuals which has been used to describe two distinct groups of women [29]. Interestingly, these two types of individuals are associated with different habitual intakes, the marginally nourished and the very well nourished. For years some researchers in the field of obesity research have maintained the idea that individuals exist who are capable of consuming prodigious amounts of food yet remain lean. It is possible, therefore, that the HF and LF may constitute a useful investigative approach for examining the relationship between energy intake and energy utilisation [30].

\section{Experimental approach}

The functional phenotype approach, as applied here, is designed to limit the number of possible variables that could potentially influence susceptibility. Even restricting the study to individuals habitually consuming a particular diet, the situation remains complicated, but the dietary contribution to this complexity is largely eliminated. In a comparison of high- and low-fat phenotypes (individuals habitually consuming a $43 \%$ or greater fat diet, or $32 \%$ or less fat diet), there was a greater number of obese people (BMI>30) among the high-fat phenotypes. However, a significant, and quite surprising, feature of this study of fat intake, appetite and BMI [24] was the distribution of BMIs among the high-fat phenotypes. The range of BMI was 17-42 (after under-reporters had been excluded). This indicated that, although habitual high-fat consumption was associated with obesity (and probably constituted a risk factor for weight gain), some individuals resisted weight gain and remained lean (Fig. 2). This clearly indicates that the relationship between weight gain and dietary fat does not constitute a biological inevitability.

The design in our approach involves comparing individuals from different ends of the distribution of BMI.

In our original use of the functional phenotype approach $[25,27]$, we compared people with quite different habitual diets (high-fat vs. low-fat) but with very similar BMIs. Here, we have compared individuals with very similar habitual diets (high-fat) but with quite different BMIs.

In order to initiate the comparison we have analysed data from very large surveys of nutrition and weight (e.g., [31]) in order to provide fundamental information. Table 2 shows some characteristics of individuals identified as habitual high-fat consumers by dual criteria of a high absolute fat intake and a high-energy percent of fat. These data can be regarded as reasonably valid because low-energy reporters have been excluded. The data for men and women show small groups of individuals with very high habitual fat intakes but with divergent BMIs - the susceptible individuals with BMIs of 31.2 (male) and 32.9 (female) and the resistant with BMIs of 23.3 and 21.5 , respectively. The average percent fat intakes were about $45 \%$ and the absolute fat intakes about $160 \mathrm{~g}$ and $115 \mathrm{~g}$ for men and women, respectively. Given this similarity in habitual fat intakes, why are some of these individuals lean and others obese? One clue is apparent in the average daily energy intakes, which indicate that the susceptible appear to be eating approximately $0.5 \mathrm{MJ}$ of food energy per day more than the resistant subjects. Using an approximate formulation for

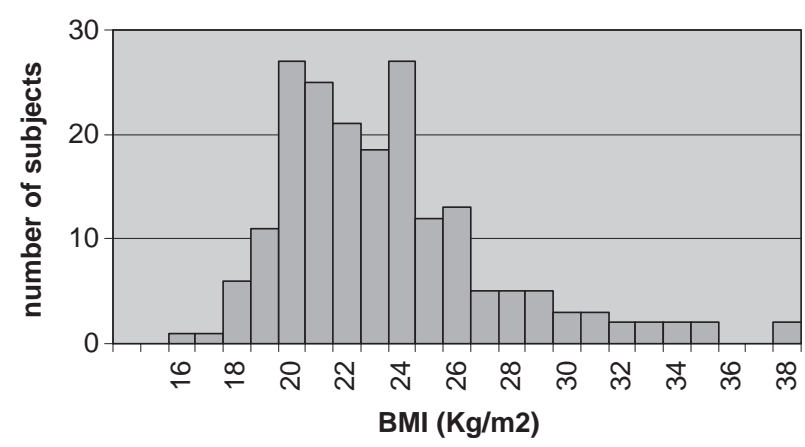

Fig. 2. Frequency distribution of BMI for habitual high-fat consumers (phenotypes) defined by the absolute daily amount of fat consumed [24]. 
Table 2

Data from the dietary and nutritional survey of British adults (1986-1987) for 2188 adults completing 7-day weighed food intakes

\begin{tabular}{lrlllll}
\hline Phenotype & \multicolumn{1}{c}{$n$} & Prevalence $(\%$ of data set) & BMI $\left(\mathrm{kg} / \mathrm{m}^{2}\right)$ & Energy intake $(\mathrm{MJ} /$ day $)$ & Fat intake $(\mathrm{g} /$ day $)$ & Fat intake $(\%$ energy) \\
\hline Resistant male & 18 & 0.8 & $23.3(1.5)$ & $12.8(1.7)$ & $156(19.6)$ & $45.0(2.0)$ \\
Susceptible male & 7 & 0.3 & $31.2(3.2)$ & $13.2(1.3)$ & $162(19.7)$ & $44.9(2.0)$ \\
Resistant female & 39 & 1.8 & $21.5(1.9)$ & $9.0(1.0)$ & $112(11.7)$ & $45.7(2.8)$ \\
Susceptible female & 10 & 0.5 & $32.9(5.7)$ & $9.5(1.1)$ & $115(8.9)$ & $44.8(4.1)$ \\
\hline
\end{tabular}

Subjects identified as mis-reporters (EI/BMR ratio $<1.3$ ) have been excluded. The subjects were classified according to a dual criterion of $\%$ fat consumed and also the absolute weight of fat. Male high-fat consumers: $>43 \%$ fat and $>120 \mathrm{~g} /$ day; female high-fat consumers: $>43 \%$ fat and $>85 \mathrm{~g} /$ day. DIO-R $=$ resistant subjects, DIO-S $=$ susceptible subjects. Values in parentheses represent means (S.E.).

relating energy intake to weight gain, this difference if maintained every day would amount to a weight gain of approximately $12 \mathrm{lbs}$ or $5 \mathrm{~kg}$ per year. In turn, if this was maintained for a period of 6 to 7 years (and not compensated for by changes in other parts of the energy balance system), it could account for the difference in BMIs observed. If this interpretation is correct, the question to be posed is: what mechanisms account for the elevation of daily energy intake (by approx. $125 \mathrm{kcal}$ ) in the susceptible people compared with the resistant? Of course, it has to be considered that the differences observed in the BMIs of resistant and susceptible people may have been achieved via differences in energy expenditure; the differences in estimated energy intake may reflect the energy requirements for weight maintenance.

\section{Dual assessment: laboratory and natural environment}

The methodology was based on two different procedurestermed probe days and free-living days. In the probe days, subjects were intensively investigated within the laboratory, during which time they received test meal challenges during full-day exposures to either high-fat or low-fat foods, completed profiles of ratings for hunger, fullness and other sensations, performed food preference and forced-choice food selection tests and made hedonic ratings of foods consumed. The food preference checklist has previously been shown to be sensitive to the effects of interventions on choice of specific food categories. The logic underlying this strategy was to detect any differences between susceptible and resistant individuals in the strength of satiation or in post-ingestive satiety to high-fat or high-carbohydrate (low-fat) foods (with weight and energy density held constant). The tools were also designed to reveal differences in hedonic responsiveness to high-fat and low-fat foods, and in the intensity of liking and wanting to foods in general.

Indirect calorimetry was also used to determine RMR, RQ and other parameters related to energy expenditure and fuel utilisation. Certain cohorts of subjects also underwent structured interviews on matters relating to eating and food choice. The transcripts of the interviews were subsequently subjected to content analysis to reveal the major themes used by susceptible and resistant individuals when describing their own eating habits. A number of questionnaires including TFEQ [19], DEBQ [32] and EAT-26 [33] were administered in order to disclose underlying traits and symptoms characterising eating activities and the motivation to eat.
On free-living days, subjects recorded all foods consumed in structured diaries following intensive instruction from the study dietician. Daily profiles of hunger, fullness and the other sensations were captured through periodic ratings using the EARS - an electronic data capture device for visual analogue ratings [34,35]. The intention behind the dual assessment methodology was to assess behavioural processes related to risk factors for over-consumption in the laboratory and under more natural free-living conditions. The approach was also designed to investigate differences between susceptible and resistant individuals in both homeostatic (hunger and satiety responses) and hedonic components of eating. An important further feature was to reveal any differences in states and traits related to food intake control and the potential for over-consumption.

All subjects studied were assessed as habitual high-fat consumers using the tools of FFQ, DINE and SFQ (see [25]) -all designed to yield an estimate of fat intake-and subsequently by monitored food diary recording. The identity of high-fat consumers was based on the percent fat in the diet and the total weight of fat eaten. All subjects were male and the mean ages of susceptible and resistant subjects were very similar. This matching for age was essential in order to ensure a similar length of exposure to the high-fat diet over time. Susceptible and resistant subjects were classified based on BMI, with mean values of 34.0 and 22.3 , respectively.

\section{Experimental outputs}

The results of the investigation indicate that four or more characteristic features could account for an elevated energy intake in susceptible people. First, the probe day nutritional challenges indicated a specific post-ingestive response to highfat, but not to low-fat, challenges in susceptible people. Although susceptible subjects had higher daily energy intakes than resistant for both high-fat and low-fat probe days (expected because of the greater body weight of susceptible subjects), the high-fat foods brought about a significantly weaker suppression of hunger in the post-ingestive period, and this occurred although the high- and low-fat meals were matched for energy, weight and energy density. The individual subject profiles for the high-fat and low-fat challenge meals are shown in Fig. 3.

This weak effect was specific to the high-fat days and it did not occur during the low-fat probe days and it did not occur in resistant individuals. This may have been one reason why susceptible people consumed much larger amounts of food during the evening snacking period (data not shown). 

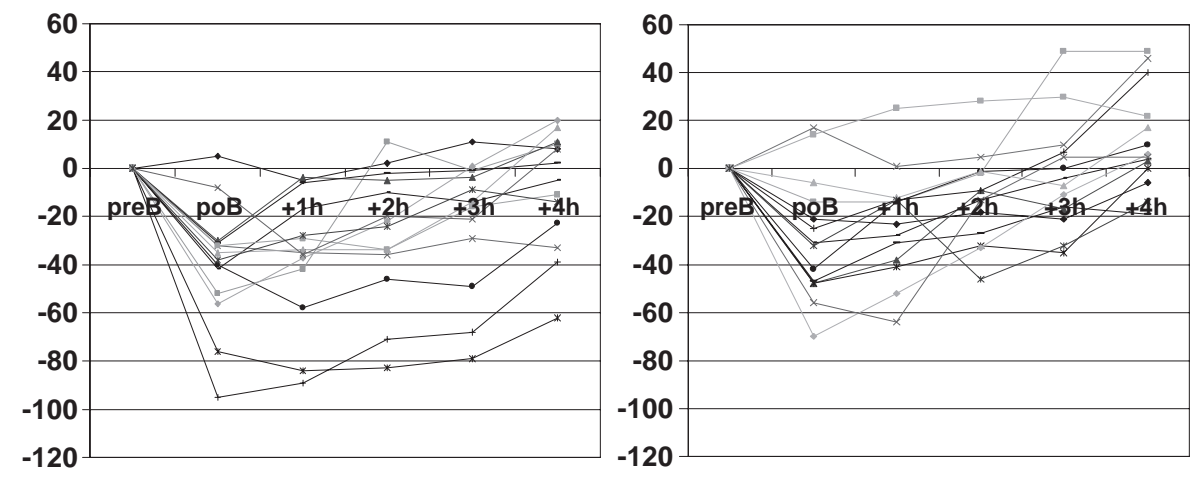

Fig. 3. Effect of low-fat (left panel) and high-fat (right panel) equi-energetic meals of equal weight on individual profiles of hunger in habitual high-fat consumers susceptible to weight gain (difference between conditions significant at $p<0.05$ ).

A second difference in the post-ingestive response was reflected in the pattern of hedonic preferences measured by the food preference checklists. Characteristically, subjects reliably demonstrate a preference for high-protein and high-fat foods when hungry (before a meal), with a shift in preference for lowenergy items when satiated (immediately after a meal). This pattern was clearly shown by the resistant subjects. However, as shown in Fig. 4, the susceptible individuals retained a preference for high-energy (high-fat) foods over low-energy foods in the post-ingestive satiated state.

A third difference between the susceptible and resistant subjects was clearly observed in the analysis of the TFEQ data. Although the two groups showed almost identical scores on
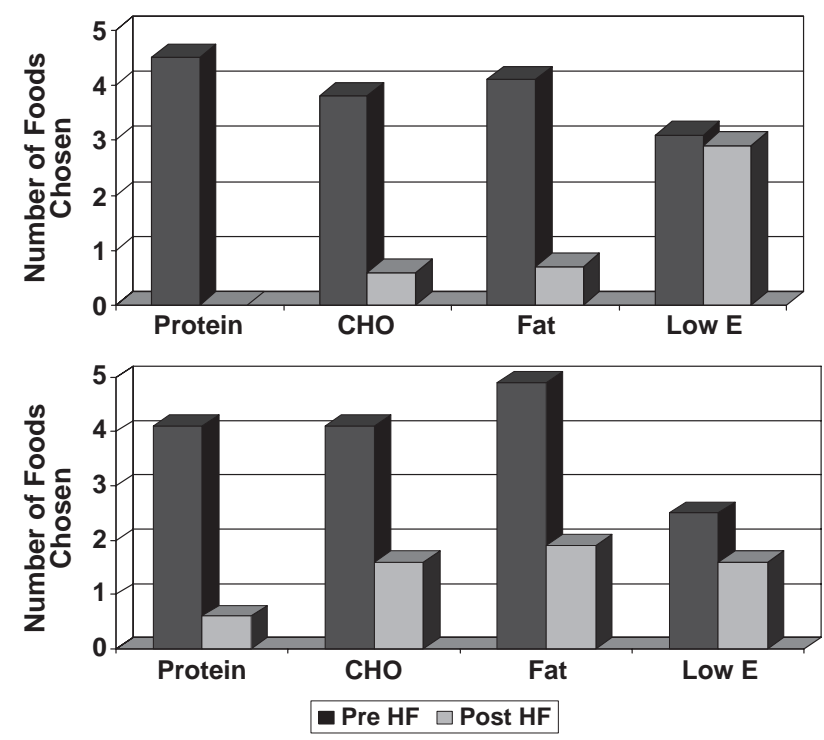

Fig. 4. Frequency of responses on the food preference checklist, before (dark columns) and after (light columns) the high- or low-fat test meals for foods high in protein, carbohydrate and fat, and for low-energy foods in habitual high-fat consumers. Top panel: resistant subjects; bottom panel: susceptible subjects. For the resistant group, in the post-ingestive state following the meal, there is a decrease in preference for protein, fat and carbohydrate foods and an increase in preference for low-energy foods. In the post-meal state, the most preferred foods are low energy. The susceptible group does not show an increase in preference for low-energy foods after the meal at which time the most preferred foods are high-fat. the Restraint factor, the susceptible group had much higher average scores for the factors of Disinhibition and Hunger [36]. There has been some discussion over whether or not these two factors constitute separate dimensions or whether they form components of one super-factor [37]. However, high scores represent a strong underlying readiness to eat in response to environmental triggers coupled with a noticeable level of hunger. This creates what we have referred to as opportunistic eating. This is clearly demonstrated by the susceptible but not the resistant subjects (Table 3).

A fourth difference was observed in the analysis of the qualitative reports of subjects when they described their own subjective feelings about their own appetites, food preferences and sensations that motivated them to eat. The difference related to the general hedonic responsiveness to foods and the intensity of the experienced sensations. Although both groups of subjects prioritized taste and enjoyment of food over concerns about health, and the enjoyment of high-fat foods, the resistant people did report that they could eat whatever they wanted to without gaining weight. Indeed some of the resistant individuals stated that they were actually trying to gain weight [38] In contrast the susceptible subjects had even stronger sensations of enjoyment related to food selection, and specifically to tastiness. Enjoyment was strongly associated with the taste of food. Strong descriptors were often used to characterise the desire for taste including statements such as 'I just love these...' or 'I just go crazy for ...'. Also reported was the enjoyment derived from eating large amounts of food and subjects admitted eating larger quantities. This feature was also apparent from the objective data collected from the probe day test meals. Whereas the resistant subjects reported that they ate less with a dysphoric mood, susceptible people said they ate more when depressed or worried [39].

Table 3

Average TFEQ scores for age-matched male resistant and susceptible subjects eating a habitual high-fat diet ( $>43 \%$ fat)

\begin{tabular}{llll}
\hline TFEQ factor & Resistant & Susceptible & $t$-Test \\
\hline Restraint & 4.3 & 4.3 & NS \\
Disinhibition & 3.1 & 8.8 & $p<0.01$ \\
Hunger & 4.1 & 7.4 & $p<0.01$ \\
\hline
\end{tabular}




\section{Summary}

Taken together the various measurable outputs from this experimental approach have identified clear differences between the susceptible and resistant subjects. These differences are apparent in both states and traits, and in the homeostatic and hedonic influence over eating. The state differences are interesting because they signify a weak post-ingestive satiety response that is specific to fat intake. The careful monitoring of peri-prandial sensations has indicated a suppression of hunger (an index of the strength of satiety) following high-fat meals. It can be speculated that this may be associated with lower levels of putative peptide satiety signals such as CCK [40] or PYY [41], both of which may be associated with fat intake. This type of action is also reminiscent of differences in compensatory sensations and behaviour in reduced-obese individuals [42]. Reduced-obese people have a tendency to re-gain weight and therefore may be a model of the susceptible individuals studied here. This weak satiety signalling response is an example of an altered response within the homeostatic control of eating.

There was also a clear indication of differing influences of hedonic responses to food. This has been illustrated previously in the stronger preferences (degree of liking) expressed by susceptible people for the high-fat foods they habitually consume [38], and by the strong taste preferences for fat demonstrated by some obese people [43]. Here the hedonic responsiveness of the susceptible individuals was demonstrated by the colourful and intense language used to describe the enjoyment derived from foods and from the eating practices itself. The compulsive nature of the strong desire for the tasting of food may turn out to have a neurochemical basis [44]. Another revealing feature of these qualitative reports was the revelation that some resistant individuals are actually trying to gain weight. These people appear to be examples of those individuals, often cited but rarely described, who also appear to be able to eat whatever they want without gaining weight. They may be examples of individuals with a 'profligate' physiology [29], although one feature of their behaviour is that they appear unable to eat beyond a certain amount despite deriving great enjoyment from food. This implies that the satiety signalling systems of resistant individuals are functioning appropriately. The strong inhibition of over-consumption (perhaps by a margin of $0.5 \mathrm{MJ} /$ day) is apparently sufficient to prevent a positive energy balance and weight gain.

In contrast to the strong post-ingestive control in resistant subjects, susceptible individuals display a fragile or vulnerable post-ingestive state. This is characterised not only by a weak satiety response to fat, but also by active maintained preference for high-fat foods over low-energy foods. Consequently, the immediate post-ingestive period is a risky time period for susceptible individuals when they appear to be vulnerable to inappropriate food choices (high-fat, high-energy). In other words, they appear to be relatively insensitive to the effects of sizeable energy loads. As noted by others, 'What may determine whether a particular individual becomes obese or not may relate to the ability of that individual to sense and respond appropriately to periods of positive energy balance' [42, p, 253].
Along with the altered 'states' of the appetite profile in susceptible people, their relationship with food is also characterised by strong 'traits' - namely Disinhibition and Hunger measured by the TFEQ. In contrast to the episodic activity of appetite states, these traits convey a tonic readiness to eat and therefore confer an enduring and constant vulnerability to be stimulated to eat. These traits are often associated with binge eating and therefore render individuals prone to periods of overconsumption [45]. There is an increasing body of evidence that the trait of Disinhibition is linked to the tendency to gain weight in various populations in the US [46] and the UK [47], and is also associated with obesity in Canadian cohorts [48]. There is also evidence that high Disinhibition scores are associated with dietary relapse [49]. Given this accumulating weight of evidence, it is not surprising that people showing susceptibility to weight gain in the present study exhibited strong levels of the traits of Disinhibition and Hunger.

The characteristics of individuals identified as susceptible to weight gain forms a cluster of risk factors, each one of which contributes a certain amount to the tendency to over-consuming at formal eating episodes (scheduled meals) or in more unorganized or informal environments. The characteristics which include weak post-ingestive satiety, heightened hedonic responsiveness and strong oro-sensory experiences map very well onto the model of risk-factors set out in Table 1 (see early part). The model therefore has empirical validation. It should be kept in mind that we have emphasised here risk factors in the field of appetite control, but it is clear that susceptible individuals also display reduced energy expenditure [36] and this can be taken into account when describing routes to obesity [30]. Importantly, the identification of risk factors for overconsumption that characterise individuals susceptible to weight gain can be used to devise procedures-behavioural, psychological, nutritional or pharmacological-that can be employed to combat specific features of vulnerability. The fact that susceptibility to weight gain is characterised by a cluster of homeostatic and hedonic risk factors suggests a generally 'conservative' biological substrate underlying these patterns. This proposed genetic profile of allelic variation will map onto a phenotypic profile of states and traits. It can be expected that the features of susceptibility-genotypic and phenotypic-will display considerable diversity. The characteristics of susceptible individuals disclosed here could define the profile of a 'thrifty phenotype'.

\section{Acknowledgements}

The work described above was funded by the European Commission, Framework V, Quality of Life and Management of Living Resources Programme. Number: QLK1-CT2000-00515.

\section{References}

[1] Ravussin E, Gautier JF. Metabolic predictors of weight gain. Int J Obes 1999;23:37-41. 
[2] Ravussin E, Kozak LP. Energy homeostasis. In: Hofbauer KG, Keller U, Boss O, editors. Pharmacotherapy of obesity — options and alternatives. Boca Raton: CRC Press; 2004. p. 488.

[3] Barsh GS, Farooqui IS, O'Rahilly S. Genetics of body weight regulation. Nature 2000;404:644-51.

[4] Marti A, Moreno-Aliaga MJ, Hebebrand J, Martinez MJ. Genes, lifestyles and obesity. Int J Obes 2004;28:S29-36.

[5] Farooqui IS, Keogh JM, Giles SH, Yeo EJ, Lank TC, O’Rahilly S. Clinical spectrum of obesity and mutations in the melanocortin 4 receptor gene. N Engl J Med 2003;348:1085-95.

[6] Schwartz MW, Woods SC, Porte D, Seeley RJ, Baskin DG. Central nervous system control of food intake. Nature 2000;404:661-71.

[7] Halford JCG, Rodgers RJ, Ishii Y, Blundell JE. The psychopharmacology of appetite: targets for potential anti-obesity agents. Curr Med Chem-Cent Nerv Syst Agents 2003;3:283-310.

[8] De Graaf C, Blom AM, Smeets PAM, Stafleu A, Hendriks HFJ. Biomarkers of satiation and satiety. Am J Clin Nutr 2004;79:946-61.

[9] Berthoud H-R. Multiple neural systems controlling food intake and body weight. Neurosci Biobehav Rev 2002;26:393-428.

[10] Saper CB, Chou TC, Elmquist JK. The need to feed: homeostatic and hedonic control of eating. Neuron 2002;36:199-211.

[11] Blundell JE, Finlayson GS. Is susceptibility to weight gain characterised by homeostatic or hedonic risk factors for over consumption? Physiol Behav 2004;82:21-5.

[12] Blundell JE, Le Noury J. Appetite control and palatability of food in humans: does the pleasure of eating lead to obesity. In: Medeiros-Neto G, Halpern A, Bouchard C, editors. Progress in Obesity Research, vol. 9. 2003. p. $822-5$.

[13] Lowe MR, Levine AS. Eating motives and the controversy over dieting: eating less than needed versus less than wanted. Obes Res 2005;13: $797-806$.

[14] Berridge KC. Food reward: brain substrates of wanting and liking. Neurosci Biobehav Rev 1996;20:1-25.

[15] Berthoud H-R. Neural control of appetite: cross talk between homeostatic and non-homeostatic systems. Appetite 2004;43:315-31.

[16] Hill AJ, Blundell JE. Model system for investigating the actions of anorectic drugs: effect of D-fenfluramine on food intake, nutrient selection, food preferences, meal patterns, hunger and satiety in healthy human subjects. Adv Biosci 1987;60:277-389.

[17] Yeomans MR, Wright P. Lower pleasantness of palatable foods in nalmefene-treated human volunteers. Appetite 1991;16:249-59.

[18] Blundell JE. What foods do people habitually eat? A dilemma for nutrition, an enigma for psychology. Am J Clin Nutr 2000;71:3-5.

[19] Stunkard AJ, Messick S. The Three Factor Eating Questionnaire to measure dietary restraint, disinhibition, and hunger. J Psychosom Res 1985;29:71-81

[20] Neel JV. Diabetes mellitus: a thrifty genotype rendered detrimental by progress? Am J Hum Genet 1962;14:353-63.

[21] Drewnowski A. The behavioral phenotype in human obesity. In: Capaldi ED, editor. Why we eat what we eat: the psychology of eating. Washington (DC): American Psychological Association; 1996. p. 339.

[22] Blundell JE, Lawton CL, Cotton JR, Macdiarmid JI. Control of human appetite: implications for the intake of dietary fat. Am J Clin Nutr 1996;16:285-319.

[23] Blundell JE, Cooling J. High-fat and low-fat (behavioural) phenotypes: biology or environment? Proc Nutr Soc 1999;58:773-7.

[24] Macdiarmid JI, Cade JE, Blundell JE. High and low fat consumers, their macronutrient intake and body mass index: further analysis of the National Diet and Nutrition Survey of British Adults. Eur J Clin Nutr 1996;50: $505-512$.

[25] Cooling J, Blundell JE. Are high-fat and low-fat consumers distinct phenotypes? Differences in the subjective and behavioural response to energy and nutrient challenges. Eur J Clin Nutr 1998;52:193-201.

[26] French SJ, Murray B, Rumsay RDE, Fadzlin R, Read NW. Adaptation to high fat diets: effects on eating behaviour and CCK. Br J Nutr 1995; 73:179-89
[27] Cooling J, Blundell JE. Differences in energy expenditure and substrate oxidation between habitual high fat and low fat consumers (phenotypes). Int J Obes 1998;22:612-8.

[28] Cooling J, Barth J, Blundell JE. The high-fat phenotype: is leptin involved in the adaptive response to a high fat (high energy) diet? Int J Obes 1998; $22: 1132-5$.

[29] Goldberg GR. From individual variation in energy intakes to variations in energy requirements and adaptations to them. Br J Nutr 1997;78:81-94.

[30] Blundell JE, Cooling J. Routes to obesity: phenotypes, food choices and activity. Br J Nutr 2000;83:S33-8.

[31] Gregory J, Foster K, Tyler K, Wiseman M. The dietary and nutritional survey of British adults. London: HMSO; 1990

[32] Van Strien T, Fritjers JER, Bergers GPA, Defares PB. Dutch eating behaviour questionnaire for assessment of restrained, emotional and external eating behaviour. Int J Eat Dis 1986;5:295-315.

[33] Garner DM, Garfinkel PE. The eating attitude test: an index of the symptoms of anorexia nervosa. Psychol Med 1979;9:1-7.

[34] King NA, Lawton CL, Delargy HJ, Smith FC, Blundell JE. The electronic appetite rating system (EARS): a portable computerised method for continuous automated monitoring of motivation to eat and mood. In: Wellman PJ, Hoebel BG, editors. Ingestive behavior protocols, society for the study of ingestive behavior, p. 71-6

[35] Stubbs RJ, Hughes DA, Johnstone AM, Rowley E, Reid CA, Elia M, et al The use of visual analogue scales to assess motivation to eat in human subjects: a review of their reliability and validity with an evaluation of new hand-held computerized systems for temporal tracking of appetite ratings. Br J Nutr 2000;84:405-15.

[36] Lawton Clare, Croden Fiona, Alam Rahul, Golding Cheryl, Whybrow Stephen, Stubbs James, et al. Differences between individuals resistant (DIOR) and susceptible (DIOS) to weight gain on a high fat (HF) diet. Int J Obes 2004;28(Suppl. 1):S218.

[37] Karlsson J, Persson L-O, Sjostrom L, Sullivan M. Psychometric properties and factor structure of the Three-Factor Eating Questionnaire (TFEQ) in obese men and women. Results from the Swedish Obese Subjects (SOS) Study. Int J Obes 2000;24:1715-25.

[38] Le Noury Joanna, Lawton Clare, Stubbs James, Whybrow Stephen, Blundell John. Is hedonic response a risk factor for overeating in individuals susceptible to weight gain. Int J Obes 2004;28(Suppl. 1):218.

[39] Le Noury JC. Food choice phenotypes: causes and consequences of habitual food selection. PhD thesis, University of Leeds, 2004.

[40] Greenough A, Cole G, Lewis J, Lockton JA, Blundell JE. Untangling the effects of hunger, anxiety, and nausea on energy intake during intravenous cholecystokinin octapeptide (CCK-8) infusion. Physiol Behav 1998;65(2): $303-310$.

[41] Batterham RL, Bloom SR. The gut hormone peptide YY regulates appetite. Ann N Y Acad Sci 2003;994:162-8.

[42] Cornier M-A, Grunwald Gary K, Johnson SL, Bessesen DH. Effects of short-term overfeeding on hunger, satiety and energy intake in thin and reduced-obese individuals. Appetite 2004;43:253-9.

[43] Mela DJ, Sacchetti DA. Sensory preferences for fats: relationships with diet and body composition. Am J Clin Nutr 1991;53:908-15.

[44] Volkov ND, Wise RA. How can drug addiction help us to understand obesity? Nat Neurosci 2005;8:555-60.

[45] Gillett A. Experimental and conceptual aspects of hunger and its role in eating behaviour in lean and obese humans. $\mathrm{PhD}$ thesis, University of Leeds, 2005

[46] Hays NP, Bathalon GP, McCrory MA, Roubenoff R, Lipman R, Roberts SB. Eating behavior correlates of adult weight gain and obesity in healthy women aged 55-65 years. Am J Clin Nutr 2002;75(3):476-83.

[47] Dykes J, Brunner EJ, Martikainen PT, Wardle J. Socioeconomic gradient in body size and obesity among women: the role of dietary restraint, disinhibition and hunger in the Whitehall II study. Int J Obes 2004;28(2): $262-268$

[48] Provencher V, Drapeau V, Tremblay A, Depres J-P, Lemieux S. Eating behaviors and indexes of body composition in men and women from the Quebec family study. Obes Res 2003;11:783-92. 